\title{
NONCIRRHOTIC PORTAL HYPERTENSION IN A PATIENT WITH JUVENILE SYSTEMIC LUPUS ERYTHEMATOSUS: A CASE REPORT
}

\author{
Rafael Fusaro Aguiar Oliveira ${ }^{1, \star}$, Anna Carolina Faria Moreira Gomes Tavares ${ }^{1}$, Lucas Moyses Carvalho de Oliveira ${ }^{1}$, Matheus \\ Fonseca Cardoso ${ }^{1}$, Ana Paula Bernardes Real ${ }^{1}$
}

1.Universidade Federal de Minas Gerais, Belo Horizonte (MG), Brazil.

*Corresponding author: fusarorafael@gmail.com

\section{BACKGROUND}

Noncirrhotic portal hypertension (NCPH) is characterized by the development of portal hypertension in the absence of cirrhosisdefining findings. It is a clinical manifestation found in immune-mediated and hematological diseases, such as systemic lupus erythematosus (SLE), rheumatoid arthritis, systemic sclerosis, antiphospholipid syndrome and myeloproliferative diseases. In this report, we describe a rare case of association of juvenile SLE with noncirrhotic portal hypertension.

\section{CASE REPORT}

A 27-year-old female patient has been diagnosed with SLE for 11 years. Five months ago, she started to experience periumbilical abdominal pain and bloating and in the last month, increased abdominal volume and dyspnea on exertion. On physical examination, she had a painless, ascitic and distended abdomen and splenomegaly. Upon laboratory evaluation, ascitic fluid analysis was suggestive of portal hypertension; she had normal liver and canalicular enzymes and negative serology for viral and autoimmune hepatitis. Endoscopy showed medium and large-caliber varicose veins and portal hypertensive gastropathy. Abdominal ultrasonography showed the liver with normal shape, size and echogenicity; however, a partial, nonocclusive thrombus in the right branch of the portal vein was shown. Antiphospholipid antibody testing was positive on this occasion. A liver biopsy was performed, and showed preserved lobular architecture, but with the presence of nodules not delimited by connective tissue. In the portal tracts, the portal venules showed decreased caliber. Such findings are suggestive of obliterative portal venopathy and rule out cirrhosis. Ascites improved with diuretic therapy, and the patient was discharged.

\section{CONCLUSION}

Around 30 cases of NCPH in association with SLE have been described in the literature. The pathogenesis of this condition appears to be related to portal blood flow abnormalities caused by chronic or recurrent infections, injury from the use of medications such as azathioprine and cyclophosphamide, hypercoagulable states, genetic predisposition, and autoimmune diseases, including SLE. In this case, the suggested mechanism is endothelial injury by autoantibodies with local hypercoagulability. Additionally, anticardiolipin antibodies could cause portal vasculopathy. This set of aggressions culminates in porto-sinusoidal vascular disease, a term that groups histologic conditions such as "obliterative portal venopathy", regenerative nodular hyperplasia", "hepatoportal sclerosis", "noncirrhotic portal fibrosis" and "incomplete septal fibrosis" (Figure 1), diseases in the small branches of the portal vein. The described case substantiates these hypotheses, as the patient had active SLE, positive antiphospholipid antibody, and had a thrombotic event.

\section{KEYWORDS}

Systemic lupus erythematosus, Noncirrhotic portal hypertension, Obliterative portal venopathy, Ascites. 


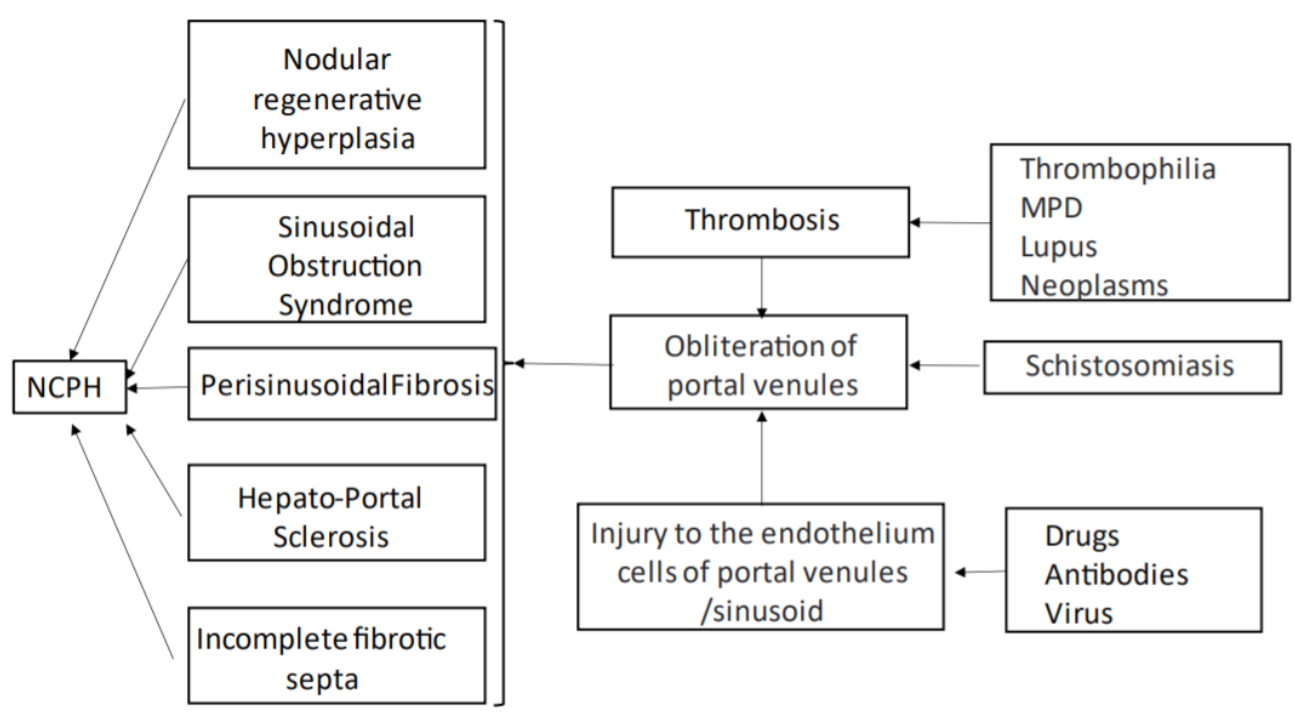

NCPH: noncirrhotic portal hypertension; MPD myeloproliferative diseases. Adapted from Hartleb M. et al.

Figure 1. Adaptative changes causing noncirrhotic portal hypertension. 